\title{
Ultralow thermal conductivity via topological network control of vibrational localization in amorphous chalcogenides
}

\author{
Kiumars Aryana \\ University of Virginia \\ Derek Stewart \\ Western Digital https://orcid.org/0000-0001-7355-2605 \\ John Gaskins \\ University of Virginia https://orcid.org/0000-0001-8622-5902 \\ Joyeeta Nag \\ Western Digital (United States) \\ John Read \\ Western Digital (United States) \\ David Olson \\ University of Virginia \\ Michael Grobis \\ Western Digital (United States) \\ Patrick Hopkins ( $\square$ peh4v@virginia.edu ) \\ University of Virginia https://orcid.org/0000-0002-3403-743X
}

\section{Article}

Keywords: amorphous chalcogenides, ultralow thermal conductivity

Posted Date: January 18th, 2021

DOI: https://doi.org/10.21203/rs.3.rs-143031/v1

License: (1) (1) This work is licensed under a Creative Commons Attribution 4.0 International License. Read Full License

Version of Record: A version of this preprint was published at Nature Communications on May 14th, 2021. See the published version at https://doi.org/10.1038/s41467-021-22999-z. 


\title{
Ultralow thermal conductivity via topological network control of vibrational localization in amorphous chalcogenides
}

\author{
K. Aryana, ${ }^{1}$ D. A. Stewart, ${ }^{2}$ J. T. Gaskins, ${ }^{1}$ J. Nag, ${ }^{2}$ J. C. Read, ${ }^{2}$ D. H. Olson, ${ }^{1}$ M. K. Grobis, ${ }^{2}$ and P. E. \\ Hopkins ${ }^{1,3,4, \text { a) }}$ \\ 1) Department of Mechanical and Aerospace Engineering, University of Virginia, Charlottesville, VA 22904, \\ USA \\ ${ }^{2)}$ Western Digital Corporation, San Jose, CA 95119, USA \\ ${ }^{3)}$ Department of Materials Science and Engineering, University of Virginia, Charlottesville, VA 22904, \\ USA \\ 4) Department of Physics, University of Virginia, Charlottesville, VA 22904, USA
}

\begin{abstract}
Amorphous chalcogenide alloys are key materials for data storage and energy scavenging applications due to their large non-linearities in optical and electrical properties as well as low vibrational thermal conductivities. Here, we report on a mechanism to suppress the thermal transport in a representative amorphous chalcogenide system, silicon telluride (SiTe), by nearly an order of magnitude via systematically tailoring the cross-linking network among the atoms. As such, we experimentally demonstrate that in fully dense amorphous SiTe the thermal conductivity can be reduced to as low as $0.10 \pm 0.01 \mathrm{~W} \mathrm{~m}^{-1} \mathrm{~K}^{-1}$ for high tellurium content with a density nearly twice that of amorphous silicon. Using ab-initio simulations integrated with lattice dynamics, we attribute the ultralow thermal conductivity of SiTe to the suppressed contribution of extended modes of vibration, namely propagons and diffusons. This leads to a large shift in the mobility edge - a factor of five - towards lower frequency and localization of nearly $42 \%$ of the modes. This localization is the result of reductions in coordination number and a transition from over-constrained to underconstrained atomic network.
\end{abstract}

In recent years, there have been numerous efforts to synthesize materials with ultralow thermal conductivities, a crucial parameter in the development of thermoelectric materials, memory devices, and thermal barrier coatings ${ }^{1-3}$. It has been generally believed that amorphous solids possess the lowest thermal conductivity possible ${ }^{4}$. The heat transport mechanisms in ultralow thermal conductivity materials are often described using formalisms originally put forth by Einstein, and later refined by others ${ }^{5-8}$, which accounts for some degree of localization of the vibrational modes or strong suppression of vibrational scattering length scales. These concepts, which partially form the basis of analytical minimum thermal conductivity models, are able to successfully predict the thermal conductivity of a wide range of amorphous solids ${ }^{7}$.

With advances in nanofabrication, several studies have shown that by reducing the mean free paths of the vibrational modes, the thermal conductivity of crystalline materials can be significantly lower than this aforementioned minimum limit $^{9-11}$. In amorphous materials, however, manipulating the atomic structure to reach values below the minimum limit is more complicated, as it is already in its highest disordered state. Thus, a question remains: how can the thermal conductivity of a fully dense amorphous solid be further reduced? One approach is to introduce chemical heterogeneity. For example, in amorphous alloys, in addition to atomic mass mismatch, disruptions in the bond structure or atomic network can significantly impede propagation of vibrational energy. Prior works have demonstrated that the thermal conductivity of amorphous thin films can be strongly reduced by breaking the number of linkers that connect the atoms through varying the stoichiometry of a given material. For instance, Ghossoub

a)Electronic mail: phopkins@ virginia.edu et al. ${ }^{12}$ showed that network connectivity in $a-\mathrm{CF}_{\mathrm{x}}$ can be manipulated by changing the fluorine concentration, resulting in nearly a factor of two reduction in thermal conductivity. Later, King et al. ${ }^{13}$ illustrated that by introducing hydrogen impurities in amorphous $\mathrm{SiC}$, the connectivity between the atoms transitions from a rigid to a percolated network, resulting in a reduction of thermal conductivity by nearly an order of magnitude. In a similar study, Braun et al. ${ }^{14}$ showed that by altering hydrogen concentration in $a-\mathrm{SiC}: \mathrm{H}$ and $a-\mathrm{SiO}: \mathrm{H}$, the thermal conductivity can be suppressed by a factor of two. In all of these studies, changing the number of bonds between constituent elements is obtained by introducing an additional impurity like fluorine or hydrogen to the baseline amorphous composition.

The insertion of these impurities not only changes the physical properties of the original material, but also introduces chemical complexities to the system. For instance, increasing the hydrogen concentration in $a-\mathrm{SiC}: \mathrm{H}$ reduces the density by more than a factor of two and also changes the nature of the bonding with the addition of hydrogen-terminated dangling bond sites. These large variations in density and bonding properties are directly proportional to the associated thermal transport, making it difficult to exclusively pinpoint the effect of bond percolation on thermal conductivity.

In this study, using different compositions of SiTe binary alloys, we demonstrate how vibrational modes can be localized to achieve ultralow thermal conductivities. According to our ab-initio molecular dynamics (AIMD) simulations, for high tellurium content SiTe, not only is the contribution of propagons to thermal conductivity subdued, but also the mobility edge shifts by a factor of five to lower frequencies, leading to localization of $42 \%$ of the modes and ultralow thermal conductivities in these amorphous alloys. Unlike previous studies where reductions in thermal conductivity follow reductions in mass density from bond termination and network- 


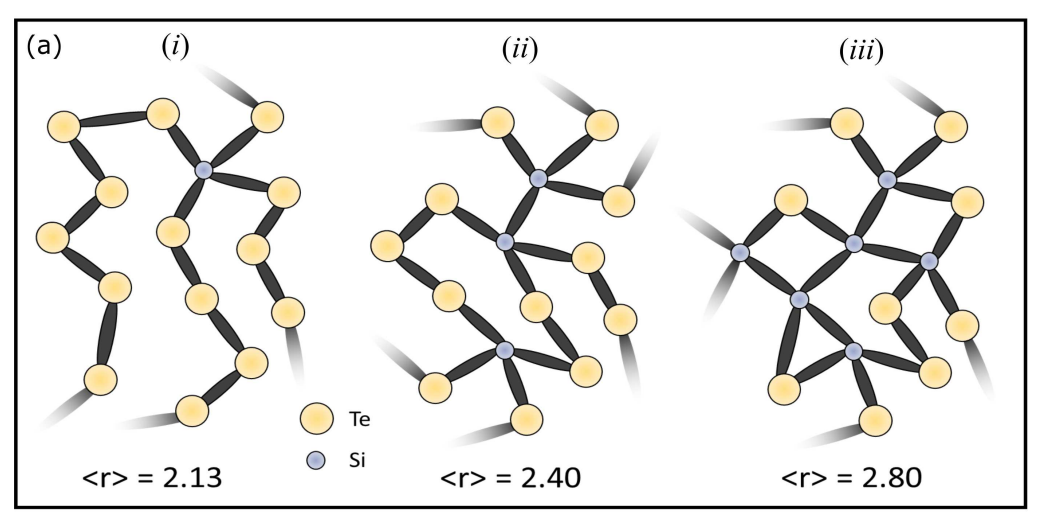

(c)
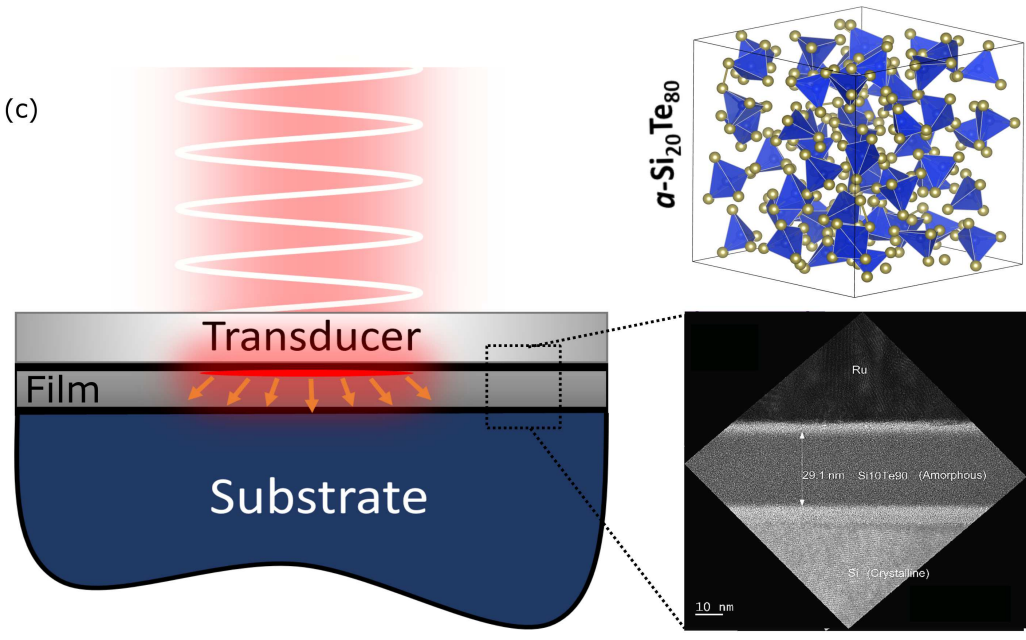
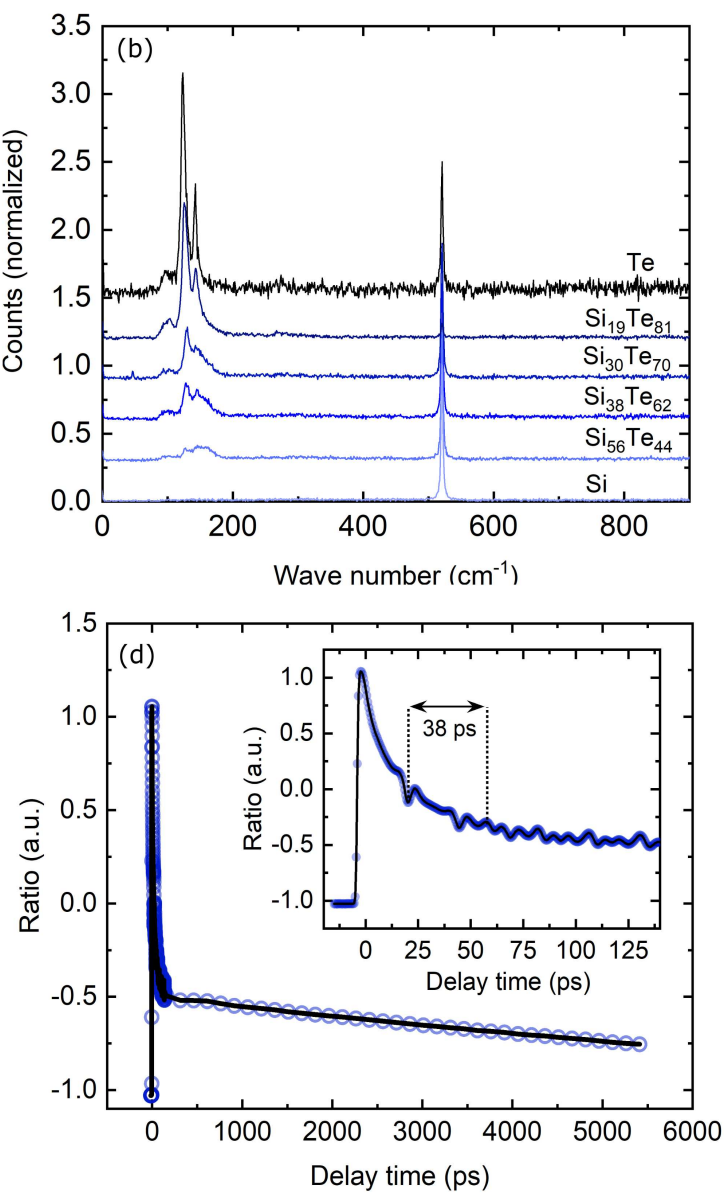

FIG. 1. (a) Schematics of amorphous networks with a total of 15 atomic sites for different Te to Si ratios representing various topological regimes: (i) under-constrained network, (ii) stress-free network, and (iii) over-constrained network. (b) Raman spectra for SiTe at different Te concentrations. The sharp silicon peak is the effect of the substrate and is not associated with the thin film properties. (c) Illustration of TDTR measurement geometry, measurement technique and the corresponding layers studied, (d) Representative TDTR data for $40 \mathrm{~nm}$ thick $a-\mathrm{Si}_{19} \mathrm{Te}_{81}$. The inset shows the picosecond acoustic measurements where the distance between the troughs and the peaks in the measured TDTR data are related to the time it takes for acoustic waves traveling at the speed of sound to transverse the $a-\mathrm{Si}_{19} \mathrm{Te}_{81}$ film.

ing, depending on the composition of the SiTe system, we can achieve ultralow thermal conductivities while increasing the mass density. This is a consequence of the differing network coordinations of silicon and tellurium and resulting vibrational localization that ensues from creating a solid solution of these two differently coordinated elements. To the best of our knowledge, our work represents the first systematic demonstration of how thermal conductivity changes across different topological network regimes: under-constrained, stress-free, and over-constrained.

In IV-VI and V-VI chalcogenide alloys, as the concentration of one constituent changes relative to another, depending on the number of covalent bonds per element, the mean atomic coordination number changes. This leads to a natural evolution of the atomic network that will directly affect the physical properties of the amorphous alloy. According to glass theory, disordered media are categorized into (i) flexible polymeric glasses consisting of long chains of randomly oriented atoms with low melting temperatures, (ii) stress-free amorphous structures with optimal glass formation proper- ties, and (iii) rigid amorphous solids consisting of a tightly interconnected network of atoms with high melting temperatures. Inspired by Maxwell's mathematical model for truss structures, Phillips ${ }^{15}$ proposed that when the number of local bonding constraints, $\mathrm{n}_{c}$, on an atom equals the number of degrees of freedom, the atomic structure is stress-free. For a three-dimensional network, this occurs when $n_{c}=3$ and the mean coordination number, $\left\langle r_{m}\right\rangle$, is 2.40 , which is generally referred to as the rigidity threshold; below this rigidity threshold, the material behaves like a polymeric glass, which is classified as under-constrained, while above this limit, the material is rigid and classified as over-constrained.

To study the effect of atomic network in amorphous materials on the thermal conductivity, we deposited thin films of silicon telluride $\mathrm{Si}_{x} \mathrm{Te}_{1-x}$ and selenium telluride $\mathrm{Se}_{x} \mathrm{Te}_{1-x}$ with different compositions via magnetron sputtering, using both co-sputtering and nano-laminate techniques, as required to achieve the desired composition. We used various spectroscopy techniques such as $\mathrm{x}$-ray fluorescence (XRF), $\mathrm{x}$ ray diffraction (XRD), Raman, and transmission electron mi- 
croscopy (TEM) to characterize the films compositions, structures, vibrational energies, and thicknesses, respectively (see supplementary note 1). The schematic in Fig. 1(a) illustrates different possibilities of network topology based on the silicon to tellurium ratio in amorphous $\mathrm{SiTe}$. It has been shown that in chalcogenide glasses, as the structure transitions from an over-constrained to an under-constrained network, additional low frequency vibrational modes emerge ${ }^{16-18}$. To detect any signature of these additional vibrational modes, we performed Raman spectroscopy on samples with different concentrations of tellurium. As presented in Fig. 1(b), in thin film SiTe, the spectra nearly look identical for all tellurium concentrations and no considerable shift in the location of the peak or emergence of additional peaks are detected.

The cross-plane thermal conductivity and longitudinal sound speed of the SiTe alloys were measured using timedomain thermoreflectance (TDTR), an optical thermometery technique that uses sub-picosecond laser pulses to excite and measure temperature excursions on the surface of materials, enabling the extraction of thermal and elastic properties of thin films ${ }^{19}$ (schematic in Fig. 1(c)). Figure 1(d) shows an exemplary TDTR thermal decay curve as a function of pump delay time for the $40 \mathrm{~nm} a-\mathrm{Si}_{19} \mathrm{Te}_{81}$ sample. The inset shows the first few picoseconds of this prototypical TDTR decay curve where the troughs and peaks correspond to the reflection of acoustic waves from the $\mathrm{Ru} / \mathrm{CN}_{x}$ and $\mathrm{CN}_{x} / \mathrm{Si}$ interfaces, respectively. By calculating the time between these "echoes" and with the knowledge of the film thicknesses obtained from TEM, we can estimate longitudinal sound speed of the SiTe films. The details of sample preparation, measurement technique, and corresponding parameters for data analysis are discussed in the supplementary note 2 .

In order to understand the effects of atomic network and coordination number on the thermal transport, we consider amorphous SiTe alloys with a variable mean coordination number $\left(2<<r_{m}><4\right)$ and amorphous SeTe alloys with a constant mean coordination number $\left(\left\langle r_{m}\right\rangle=2\right)$ at different tellurium concentrations. Figure 2(a) shows the thermal conductivity of amorphous SiTe and SeTe alloys as a function of Te atomic percentage at room temperature. In SeTe, as shown in red diamonds, the coordination numbers for both $\mathrm{Se}$ and $\mathrm{Te}$ are identical $\left\langle r_{\mathrm{Te}}\right\rangle=\left\langle r_{\mathrm{Se}}\right\rangle=2$, therefore, the relative atomic concentration does not alter the total bonding network and the thermal conductivity remains relatively constant with increasing the Te content. Thus, in spite of the existence of a large atomic mismatch between Se and Te, the changes in thermal conductivity for these under-constrained amorphous alloys are negligible. To further investigate the effect of atomic mass mismatch on thermal conductivity, we perform molecular dynamics simulations for amorphous $\mathrm{Si}$ with different concentration of heavy-Si atoms (with atomic mass similar to $\mathrm{Te}$ ). The inset in Fig. 2(a) shows the results of these simulations. Similar to what is observed in the SeTe composition, the thermal conductivity modestly decreases from $\sim 1.0 \mathrm{~W} \mathrm{~m}^{-1} \mathrm{~K}^{-1}$ in amorphous $\mathrm{Si}$ to $\sim 0.50 \mathrm{~W} \mathrm{~m}^{-1} \mathrm{~K}^{-1}$ in $a$-Si with $50 \%$ to $90 \%$ heavy-Si concentrations (see supplementary note 3 ).

In the amorphous SiTe alloys, on the other hand, the thermal conductivity of $a$-Si upon mixing with Te drops mono- tonically by almost an order of magnitude for high Te concentrations. The measured thermal conductivity for our $\sim 26 \mathrm{~nm}$ amorphous silicon film with no tellurium is analogous to values reported previously ${ }^{20}$ for films of comparable thicknesses $\left(0.8 \pm 0.2 \mathrm{~W} \mathrm{~m}^{-1} \mathrm{~K}^{-1}\right)$. As the Te concentration in SiTe increases, the thermal conductivity drops to as low as $\sim 0.10 \pm$ $0.01 \mathrm{~W} \mathrm{~m}^{-1} \mathrm{~K}^{-1}$ and stays relatively constant for Te content ranging from $70 \%$ to $90 \%$. For the pure Te film, the thermal conductivity increases to $0.23 \pm 0.04 \mathrm{~W} \mathrm{~m}^{-1} \mathrm{~K}^{-1}$. TEM micrographs as well as XRD reveal that our Te film is not amorphous, but rather polycrystalline. It is not uncommon for Te to crystallize during growth or post-processing as tellurium has a low glass-transition temperature, $\mathrm{T}_{g}$, which makes it extremely difficult to deposit in its amorphous state ${ }^{21}$. The measured thermal conductivity for our Te film is less than those reported in the literature ${ }^{22-24}$, which report in the range of 0.43-3.0 $\mathrm{W} \mathrm{m}^{-1} \mathrm{~K}^{-1}$, suggesting that some degree of disorder is present in our Te films. A comprehensive discussion regarding the structure of the films studied here supported by TEM and XRD measurements are given in the supplementary note 1.

Our cryogenic thermal conductivity measurements shown in Fig. 2(b) reveal that the thermal conductivities of pure $\mathrm{Te}$ and a-SiTe samples follow an amorphous-like trend, which is an indication of a high concentration of disorder in the films. This also explains why the thermal conductivity of the polycrystalline Te film is lower than literature values. In all of the cases studied here, the temperature dependent thermal conductivity trends follow those of the heat capacity, and plateau above the Debye temperature.

In order to understand the dramatic reduction in thermal conductivity of SiTe system upon addition of Te, we use existing theoretical approaches to calculate the minimum limit thermal conductivity derived in the context of phonons $\left(\kappa_{\min , \mathbf{P}}\right)$ and diffusons $\left(\kappa_{\min , \mathbf{D}}\right)$. According to Cahill and Pohl ${ }^{25}$, a lower limit to the thermal conductivity of materials is estimated based on the collective atomic vibrations, i.e. phonons, derived from the kinetic theory of gases:

$$
\kappa_{\min , \mathbf{P}}=1.21 k_{B} n^{2 / 3} v_{g}
$$

where $k_{B}$ is the Boltzmann constant, $v_{g}$ is the average sound velocity in the material, and $n$ is the number density. The average sound velocity can be written in terms of the longitudinal $\left(v_{L A}\right)$ and transverse $\left(v_{T A}\right)$ sound velocities as: $v_{g}^{2}=$ $\frac{1}{3}\left(v_{\mathrm{TA} 1}^{2}+v_{\mathrm{TA} 2}^{2}+v_{\mathrm{LA}}^{2}\right)$. Although this minimum limit to thermal conductivity has served as a successful approach to predict the thermal conductivity of disordered crystals and amorphous materials, several recent works have experimentally measured values well below this limit. This has motivated others to model the thermal conductivity in amorphous solids as a form of energy hopping between localized vibrational eigenstates. According to Allen and Feldman ${ }^{26}$ (AF), a large portion of heat in disordered solids is transferred by quantized vibrations that are neither localized nor propagating. These delocalized non-propagating vibrational modes, diffusons, carry heat by interactions with other vibrational modes with length 

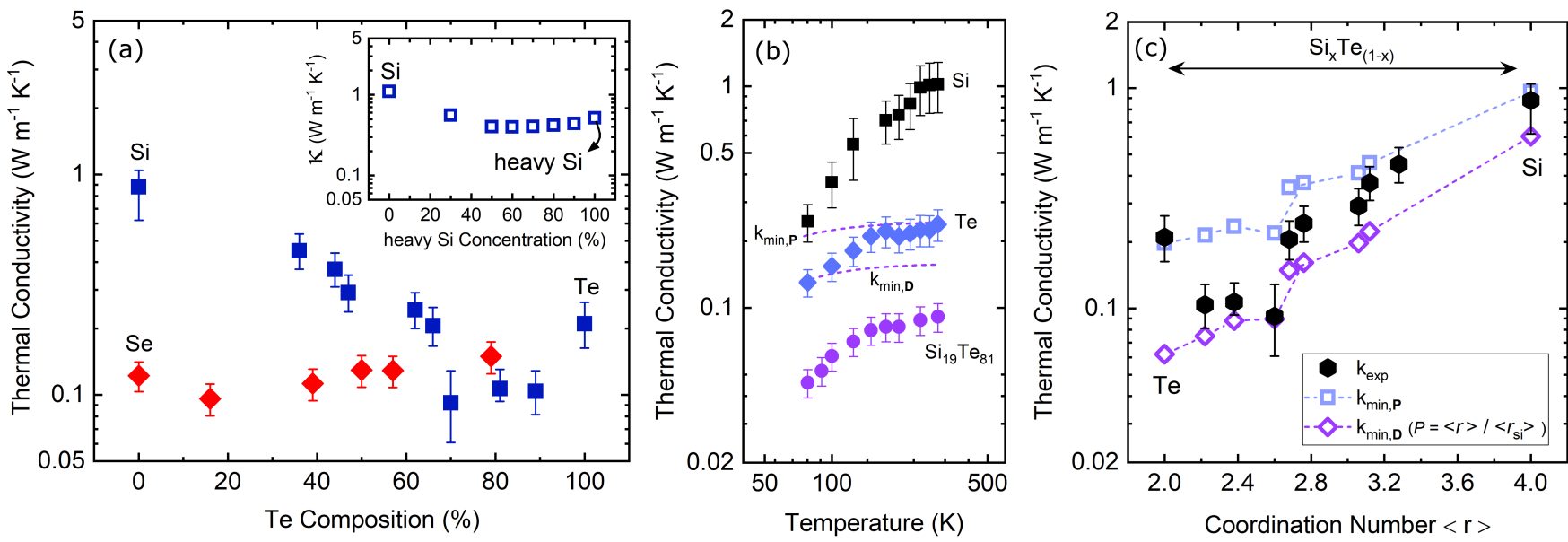

FIG. 2. (a) The measured thermal conductivity as a function of tellurium concentration in amorphous SiTe and SeTe. The inset shows the NEMD simulation results for thermal conductivity of Si (28 u), heavy Si (127 u), and their alloy. (b) Thermal conductivity of amorphous silicon, tellurium, and $\mathrm{Si}_{19} \mathrm{Te}_{81}$ as a function of temperature. For comparison, $62 \mathrm{~nm} \mathrm{WSe}$ data are included from Ref ${ }^{9}$. (c) Thermal conductivity of SiTe as a function of coordination number measured by TDTR (solid hexagons), their corresponding minimum limit (hollow squares), and the diffuson-mediated thermal conductivity (hollow diamonds).

scales on the order of the vibrational wavelength. Based on the AF formalism, Agne et al. ${ }^{7}$ suggested a modified minimum limit model for heat transport in disordered solids that relies on the concept of diffusons rather than phonons. They argued that in a disordered solid, the lower bound to thermal conductivity occurs when the thermal transport is entirely driven by diffusons. This approach, albeit the heat transfer carrier length scale being fundamentally different from those modeled in Eq. 1 , leads to a similar functional form for the thermal conductivity of disordered materials:

$$
\kappa_{\min , \mathbf{D}} \approx 0.76 P k_{B} n^{2 / 3} v_{g}
$$

where $P$ is the probability of successful energy transfer between the atoms. The dashed line in Fig. 2(b) shows the theoretical minimum limit based on phonon- and diffusonmediated thermal conductivity for $a-\mathrm{Si}_{19} \mathrm{Te}_{81}$. In the high temperature limit and maximum diffusivity where $P=1$, the calculated diffuson-mediated thermal conductivity is $\sim 37 \%$ lower than the phonon minimum limit model. Fig. 2(b) demonstrates that the measured thermal conductivity of $a$ $\mathrm{Si}_{19} \mathrm{Te}_{81}$ is well below the minimum limit calculations for both existing models. This implies that the thermal transport mechanism in $\mathrm{Si}_{19} \mathrm{Te}_{81}$ is dominated by other atomistic properties that impede the transfer of energy beyond those accounted for in the minimum limit models.

To resolve this, we revisit an assumption that was made in the diffuson-mediated thermal conductivity, which assumes $100 \%$ of attempts to transfer energy are successful between diffusons. As discussed earlier, since the coordination number in SiTe decreases by increasing the Te concentration, the alloy transitions from an over-constrained to an under-constrained network. This reduction in the number of bonds per atom eliminates the number of pathways through which diffusons can interact, and leads to a reduction in the probability of their successful energy transfer. Figure 2(c) shows the thermal conductivity of our SiTe sample as a function of coordination number. We calculate the coordination number of these alloys based on the measured relative atomic percentages assuming $\mathrm{Si}$ and $\mathrm{Te}$ as 4- and 2-coordinate elements, respectively. The result of our calculation for $\kappa_{\min , \mathbf{P}}$ and $\kappa_{\min , \mathbf{D}}$ at different coordination number are depicted in Fig 2(c) with hollow squares and diamonds. This time, for calculating $\kappa_{\min , \mathbf{D}}$, we consider variable $P$ for each alloy's configuration by normalizing their coordination number with respect to pure $\mathrm{Si}$, i.e., $P=\left\langle r_{\mathrm{m}}\right\rangle\left|\left\langle r_{\max }\right\rangle=\left\langle r_{\mathrm{Si}_{\mathrm{x}} \mathrm{Te}_{1-\mathrm{x}}}\right\rangle\right|\left\langle r_{\mathrm{Si}}\right\rangle$. Using this assumption, $P$ changes from 1 to 0.5 for $\mathrm{Si}\left(\left\langle r_{S i}\right\rangle=4\right)$ and $\mathrm{Te}\left(\left\langle r_{T e}\right\rangle\right.$ $=2$ ). By applying this condition, we calculate the diffusonmediated thermal conductivity for $\mathrm{Si}_{19} \mathrm{Te}_{81}$ as $0.09 \mathrm{~W} \mathrm{~m}^{-1}$ $\mathrm{K}^{-1}$, in better agreement with the measured values for our SiTe alloys (see supplementary note 4 ).

With the varying coordination number in SiTe with composition, these results imply that the nature of the vibrational modes contributing to thermal conductivity in these amorphous films becomes more spatially localized as the coordination number is reduced below the rigidity threshold and the SiTe atomic network transitions away from the overconstrained regime.

While the theoretical models discussed above provide reasonable qualitative insight into the thermal transport mechanisms in the amorphous SiTe alloys, we turn to ab-initio molecular dynamics and lattice dynamic simulations to gain a deeper understanding of the mechanism that drives the ultralow thermal conductivity of $\mathrm{Si}_{19} \mathrm{Te}_{81}$. In a-Si, Seyf and Henry ${ }^{27}$ showed that a small defect concentration $(\sim 10 \%)$ leads to a dramatic decrease in the population of propagons. Given the large concentration of Te in $a-\mathrm{Si}_{19} \mathrm{Te}_{81}$, we assume that propagons will have a negligible contribution to the total thermal conductivity and focus our attention on low frequency heat carrying diffusons and high frequency locons (fully lo- 

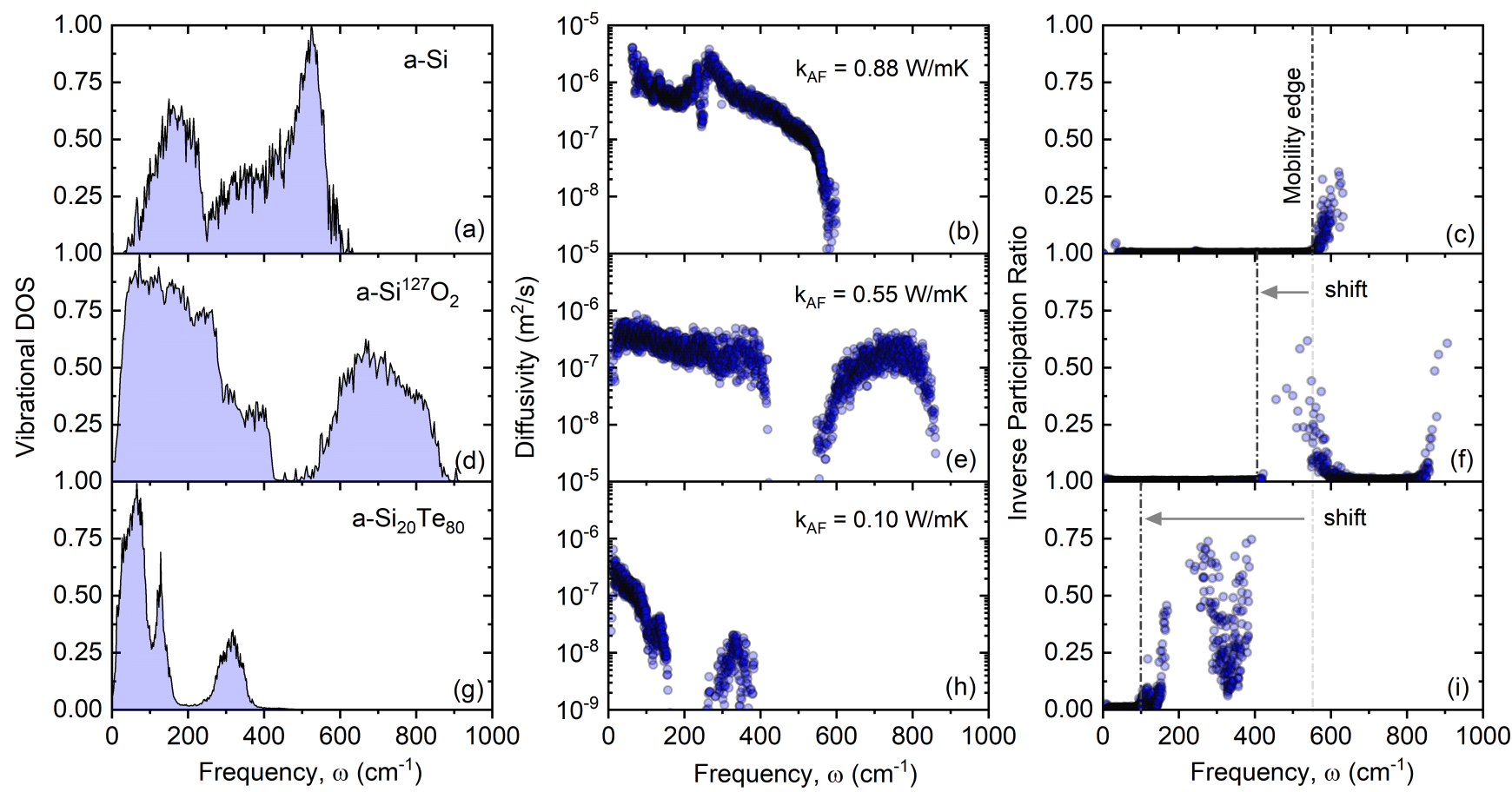

FIG. 3. (a,d,g) Vibrational density of states (DOS), (b,e,h) modes diffusivity, and (c,f,i) inverse participation ratio (IPR) for $a$-Si, $a$-Si ${ }^{127} \mathrm{O}_{2}$, and $a-\mathrm{Si}_{20} \mathrm{Te}_{80}$, respectively. The corresponding thermal conductivity are given for each system. The shift in the mobility edge with respect to the $a-\mathrm{Si}$ is depicted by an arrow.

calized modes). Locons are vibrational excitations with vibrational amplitudes that decay exponentially from the center of excitation, and normally have high frequencies. The frequency above which vibrational modes are fully localized is known as the mobility edge. The inverse participation ratio (IPR) determines the degree of localization of modes and is given by:

$$
I P R=\frac{\sum_{i}^{N}\left(\Sigma_{\alpha=1}^{3} u_{i \alpha}^{2}\right)^{2}}{\left(\sum_{i=1}^{N}\left(\Sigma_{\alpha=1}^{3} u_{i \alpha}^{2}\right)\right)^{2}}
$$

where $N$ is the number of atoms and $u_{i \alpha}$ is the eigenvector component for atom $i$ in the direction $\alpha$. For a mode that is fully localized on a single atomic site, IPR $=1$, and for a fully delocalized mode that spans all atoms, IPR $=1 / N$. While it is not possible to define a precise IPR value for the transition of modes from diffusons to locons, for this work, we define locons as modes where the eigenvector is spread across $20 \%$ or less of the atoms in the supercell (60 atoms, IPR $\geq 0.01667$ ). This convention has been used in a number of previous studies ${ }^{28-30}$ and serves as a reasonable cutoff for comparison with other works.

Figure 3(a-c) shows the vibrational density of states (DOS), mode diffusivity, and IPR for amorphous $\mathrm{Si}$, respectively. The thermal conductivity in $a-\mathrm{Si}$ is dominated by low-frequency propagons and diffusons. However, since we assume negligible contribution of propagons to thermal conductivity of SiTe films with high Te contents, we only focus on diffusons contribution which is $\sim 0.88 \mathrm{~W} \mathrm{~m}^{-1} \mathrm{~K}^{-1}$ at room temperature for $a$-Si. Based on the calculated mobility edge, we find that locons make up less than $4 \%$ of modes in $a$-Si which is in agreement with previous studies ${ }^{31}$. This indicates that almost all modes in $a$-Si are active in transferring heat. The small percentage of locons could be a result of the over-constrained bonding network in $\mathrm{a}$-Si. This means that the reduction of mean coordination number in SiTe, should increase the number of localized states.

To assess our assumption that coordination number, i.e. number of constraint on an atom, can lead to localization, we turn to another familiar amorphous structure, $a-\mathrm{SiO}_{2}$. The mean coordination number for this structure is 2.67 , which is lower than $a-\mathrm{Si}$ and based on our assumption, should have a larger percentage of locons than $a$-Si. This composition also has a similar coordination number and atomic structure to $a$ $\mathrm{SiTe}_{2}$. For instance, the $a-\mathrm{SiO}_{2}$ structure consists of randomly oriented tetrahedrons where each $\mathrm{Si}$ is bonded to 4 oxygen atoms and each tetrahedron shares an oxygen atom with another tetrahedron (Si-O-Si). Given these similarities, a major difference between $a-\mathrm{SiO}_{2}$ and $a-\mathrm{SiTe}_{2}$ is the large atomic mass difference between oxygen and tellurium. In order to facilitate comparison between $a-\mathrm{SiO}_{2}$ and $a-\mathrm{SiTe}_{2}$ alloy, we replace the oxygen mass $\left(m_{O}=16 \mathrm{amu}\right)$ with heavy-oxygen $\left(m_{T e}=127.6 \mathrm{amu}\right)$. We use a $a-\mathrm{Si}^{127} \mathrm{O}_{2}$ taxonomy for our modified $\mathrm{SiO}_{2}$ to avoid confusion with the real system.

Another notable difference between $a-\mathrm{SiO}_{2}$ and $a-\mathrm{SiTe}_{2}$ systems is the bond enthalpy which is significantly higher for $\mathrm{Si}-\mathrm{O}(\sim 450 \mathrm{~kJ} / \mathrm{mol})$ compared to $\mathrm{Si}-\mathrm{Te}(\sim 220 \mathrm{~kJ} / \mathrm{mol})$ and $\mathrm{Si}-\mathrm{Si}(\sim 225 \mathrm{~kJ} / \mathrm{mol})^{32}$. This indicates that strong $\mathrm{Si}-\mathrm{O}$ bonds would favor high frequency modes. As can be seen in 


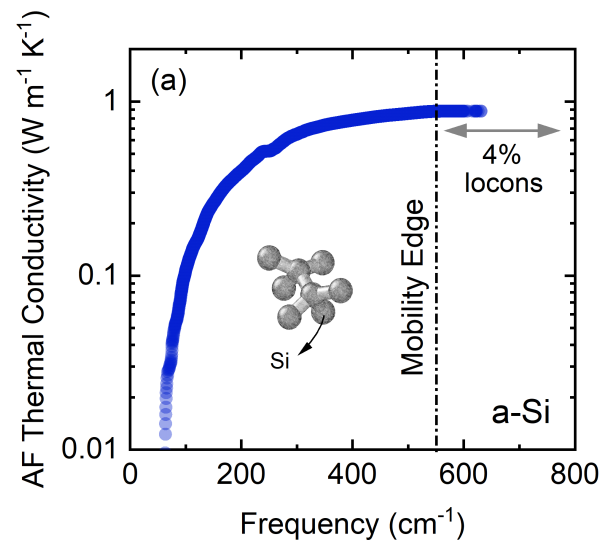

(d)

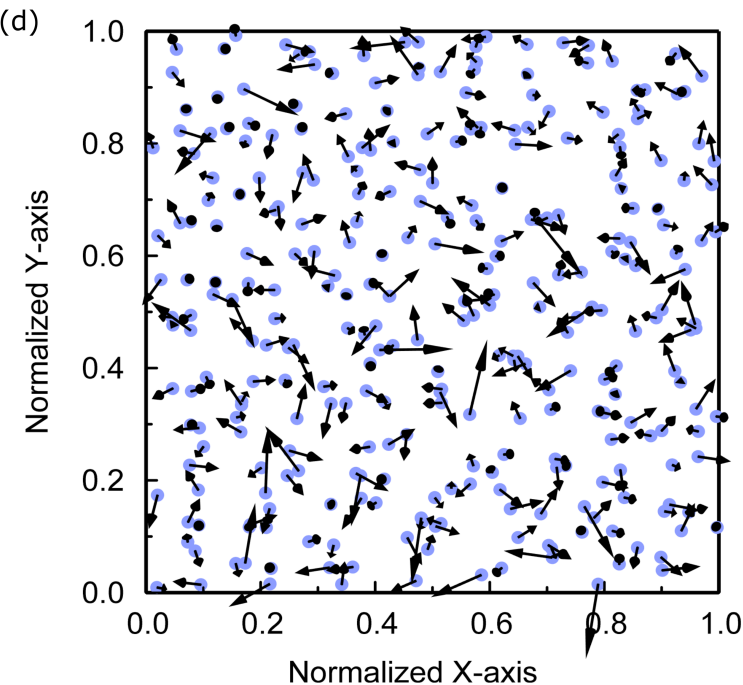

(f)

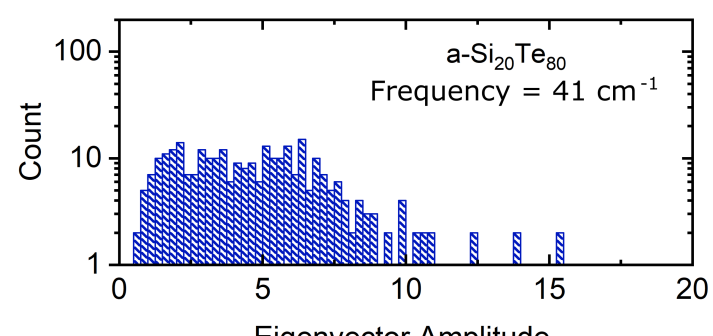

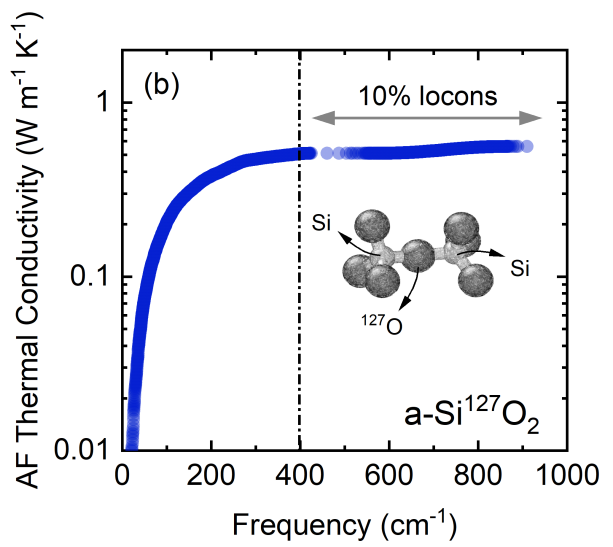
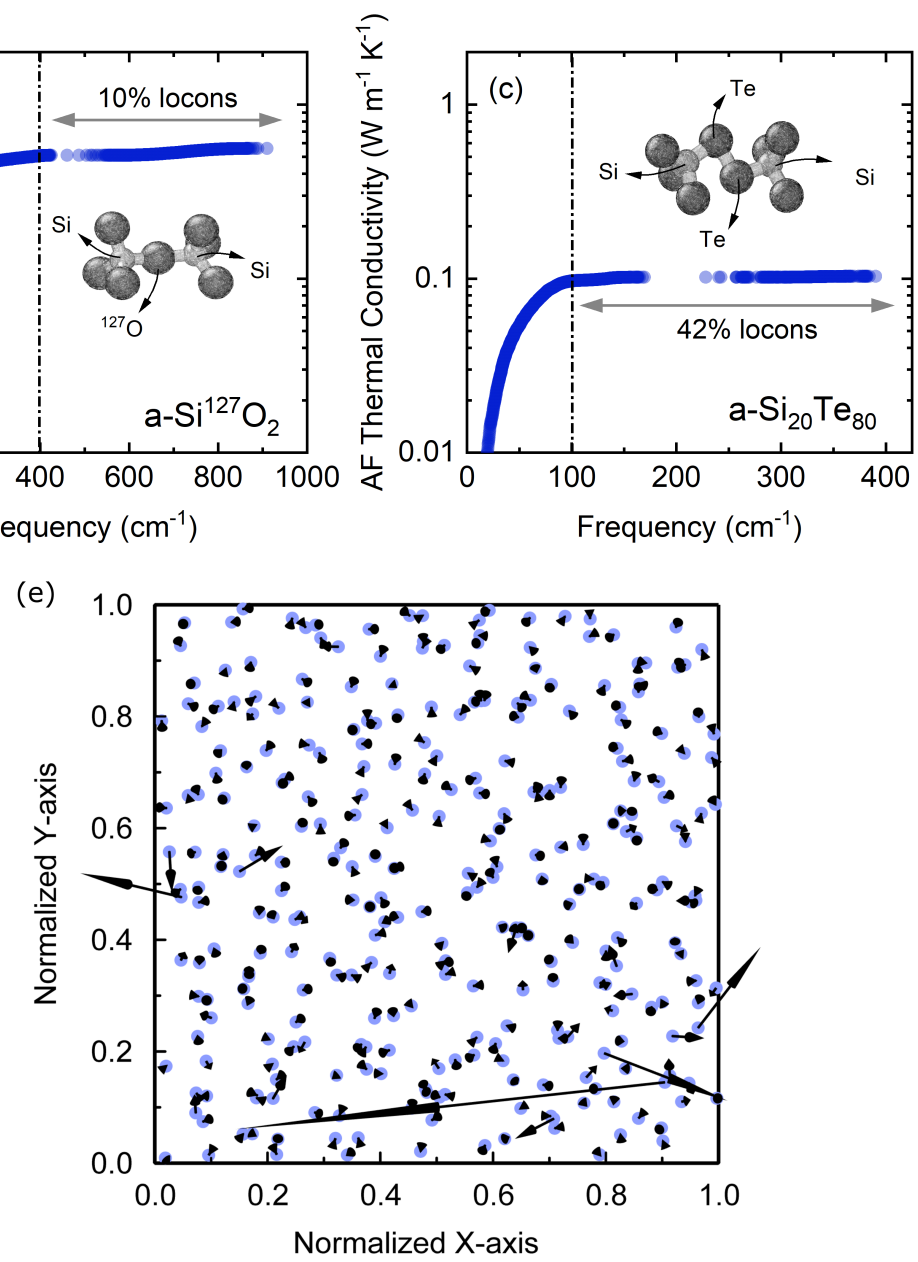

(g)

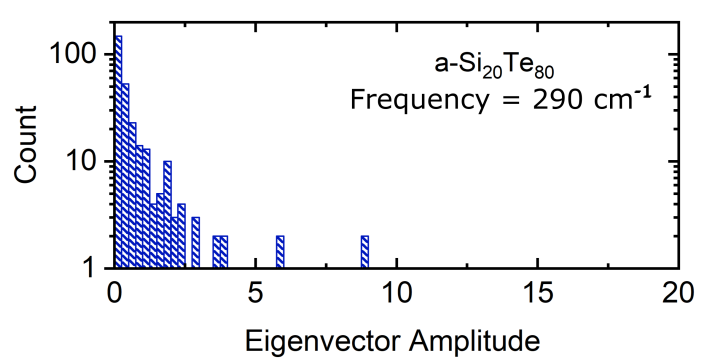

FIG. 4. Allen-Feldman thermal conductivity accumulation as a function of vibrational mode frequency for (a) $a$ - with inset showing Si-Si bond between two tetrahedrons, (b) $a-\mathrm{Si}^{127} \mathrm{O}_{2}$ with inset showing $\mathrm{Si}_{-}{ }^{127} \mathrm{O}-\mathrm{Si}$ bond between two tetrahedrons, and (c) $a$-Si $\mathrm{Si}_{20} \mathrm{Te}_{80}$ with inset showing $\mathrm{Si}-\mathrm{Te}-\mathrm{Te}-\mathrm{Si}$ bond between two tetrahedrons. (d,e) Visualization of delocalized $\left(41 \mathrm{~cm}^{-1}\right)$ and localized $\left(290 \mathrm{~cm}^{-1}\right)$ vibrational modes on either side of the mobility edge in $a-\mathrm{Si}_{20} \mathrm{Te}_{80}$. The few high amplitude eigenvectors in localized frequency are an indication of strong localization showing the energy associated with these modes are confined in a small geometric region. (f,e) Histogram indicating the population of modes based on their eigenvector amplitude at delocalized and localized frequencies.

Fig. 3(d), the $a-\mathrm{Si}^{127} \mathrm{O}_{2}$ structure has modes with frequencies higher than $a$-Si. We attribute the emergence of these higher frequency modes to the artifact of an improper interatomic potential. As a result of this, the $\mathrm{Si}^{-127} \mathrm{O}$ bonds in our modified $a$-Si ${ }^{127} \mathrm{O}_{2}$ are shorter than $\mathrm{Si}-\mathrm{Te}$, leading to the appearance of high frequency modes observed in DOS calculations. Nevertheless, these high frequency modes are localized and do not contribute to thermal conductivity. Figure 3(f) shows that the mobility edge is shifted to $\sim 400 \mathrm{~cm}^{-1}$ compared to $a-\mathrm{Si}$ and the percentage of locons has increased to $\sim 10 \%$. The $\mathrm{AF}$ thermal conductivity for $a-\mathrm{Si}^{127} \mathrm{O}_{2}$ system is estimated to be $\sim 0.55 \mathrm{~W} \mathrm{~m}^{-1} \mathrm{~K}^{-1}$.

In order to further investigate the effect of coordination number on the localization of modes, we consider $\mathrm{Si}_{20} \mathrm{Te}_{80}$ composition with a coordination number of 2.4 at the rigidity threshold. For this structure, we assume that $a-\mathrm{Si}_{20} \mathrm{Te}_{80}$ consists of randomly oriented $\mathrm{Si}$-Te tetrahedrons where the tetrahedrons do not share a $\mathrm{Te}$ atom $(\mathrm{Si}-\mathrm{Te}-\mathrm{Te}-\mathrm{Si})$. Using force 
constants derived from ab-initio calculations, we report the DOS, diffusivity and IPR for $a-\mathrm{Si}_{20} \mathrm{Te}_{80}$ alloy in Fig. 3(g-i). According to our calculations, in this composition not only is the effective frequency range reduced, but also based on our IPR calculation, nearly $42 \%$ of the modes are localized. We find that locons begin to appear for frequencies greater than $89 \mathrm{~cm}^{-1}$ and above $102 \mathrm{~cm}^{-1}$ all vibrational modes act as locons and do not contribute to thermal transport. This includes the large vibrational peak centered at $125 \mathrm{~cm}^{-1}$ due to tellurium motion and the higher optical band from $250 \mathrm{~cm}^{-1}$ to $400 \mathrm{~cm}^{-1}$ due primarily to silicon atoms. According to the IPR calculation, the mobility edge is shifted from 550 to 102 $\mathrm{cm}^{-1}$, more than a factor of five to lower frequencies. We calculate the thermal conductivity due to diffuson contribution in $a-\mathrm{Si}_{20} \mathrm{Te}_{80}$ as $0.10 \pm 0.005 \mathrm{~W} \mathrm{~m}^{-1} \mathrm{~K}^{-1}$ in excellent agreement with measured value. The uncertainty is calculated by changing the broadening factor by $50 \%$.

Figure 4(a-c) shows the thermal conductivity accumulation as a function of vibrational frequency at $300 \mathrm{~K}$. The calculated thermal conductivity for $a$-Si is comparable to those calculated by Larkin and McGaughey ${ }^{33}$. We observe that for all cases, beyond the mobility edge limit where the modes are fully localized, the thermal conductivity stays constant. This is expected since the locons contribution to total thermal conductivity is negligible. This also indicates that in $a$ $\mathrm{Si}_{20} \mathrm{Te}_{80}$, a great deal of modes $(42 \%)$ are localized leading to ultralow thermal conductivity. The delocalized and localized modes are visualized in $4(\mathrm{~d}, \mathrm{e})$ by showing the eigenvectors for modes at frequency below $\left(41 \mathrm{~cm}^{-1}\right)$ and above $\left(290 \mathrm{~cm}^{-1}\right)$ the mobility edge. The amplitude of eigenvectors for localized frequency are strongly suppressed compared to delocalized modes. The large amplitude for eigenvector observed in some of the modes are indicative of strong localization showing the energy associated with these modes are confined in a small geometric region. Figure $4(\mathrm{f}-\mathrm{g})$ indicates the population of eigenvectors based on their amplitude. According to this figure, for a frequency below the mobility edge, the amplitude of the eigenvectors are uniformly spread out from 0 to $\sim 15$. Whereas for frequency above the mobility edge, due to the effect of localization, the amplitude of a large number of modes drastically decreases to below 2 .

The thermal properties of chalcogenide materials across different topological constraint regimes have been previously investigated for bulk silicon telluride and arsenic selenide ${ }^{34,35}$. In stark contrast to the results presented in this study, the authors observe a peak for thermal diffusivity and conductivity at the rigidity threshold. Philip and Madhusoodanan ${ }^{34}$ reported a thermal diffusivity of $\sim 0.06 \mathrm{~cm}^{2} / \mathrm{s}$ for bulk a$\mathrm{Si}_{20} \mathrm{Te}_{80}$ which is more than a factor two higher than the amorphous silicon ${ }^{36}$. This could indicates that either their sample is not entirely amorphous or there is a large concentration of impurities. This discrepancy between the bulk and thin films, however, is not surprising as the defects such as impurities are common in bulk systems.

A close material cousin to $\mathrm{SiTe}$ is the well-known thermoelectric/phase-change material, GeTe, that has been extensively studied before both in terms of electrical and thermal properties. Although several studies reported the thermal con- ductivity of GeTe in amorphous phase at different tellurium concentrations $^{37-40}$, depending on the deposition process and thermometery technique, the values range from $0.1-0.23 \mathrm{~W}$ $\mathrm{m}^{-1} \mathrm{~K}^{-1}$. The absence of a unique investigation on the thermal properties of GeTe with respect to the coordination number makes it difficult to compare any trend in this composition against that of SiTe. However, due to the structural similarity between SiTe and GeTe, we use our model for $a-\mathrm{Si}_{20} \mathrm{Te}_{80}$ and replace the $\mathrm{Si}$ atomic mass with that of $\mathrm{Ge}\left(a^{-}{ }^{78} \mathrm{Si}_{20} \mathrm{Te}_{80}\right)$ and calculate its diffusivity and thermal conductivity. Since GeTe has a higher average atomic mass, one would expect to observe a lower thermal conductivity compared to SiTe. However, according to our calculations, the thermal conductivity for heavier $a^{78} \mathrm{Si}_{20} \mathrm{Te}_{80}$ does not change and remains similar to $a-\mathrm{Si}_{20} \mathrm{Te}_{80}$. This demonstrates that the low frequency heat carrying modes are not influenced by mass scattering in these unique topological chalcogenide phases.

In summary, we experimentally demonstrated that through manipulating the coordination number in amorphous silicon telluride ( $\mathrm{SiTe}$ ) alloys, the thermal conductivity can reach an ultralow value of $\sim 0.1 \mathrm{~W} \mathrm{~m}^{-1} \mathrm{~K}^{-1}$. We observe that the thermal conductivity decreases with coordination number in SiTe and reaches its minimum near the rigidity threshold where the coordination number is 2.2-2.6 with tellurium concentration of $90-70 \%$. We attribute the ultralow thermal conductivity of $a-\mathrm{Si}_{20} \mathrm{Te}_{80}$ to the strong localization of heat carrying modes evident by a large shift in mobility edge - a factor of five towards lower frequencies.

\section{METHODS}

Experimental approach. For thermal conductivity measurements we used TDTR, a pump and probe optical thermometry technique that relates the changes in the thermoreflectivity of the sample to the thermal properties. In our two-tint TDTR configuration, the output of an $80 \mathrm{MHz}$ TiSapphire femtosecond laser pulse with center wavelength of $808 \mathrm{~nm}$ is split into a pump and a probe path. The pump path is directed to an electro-optical modulator (EOM) with modulation frequency of $8.4 \mathrm{MHz}$, and the probe path is directed to a mechanical delay stage to capture the changes in temperature of the sample as a function of time. The femtosecond laser pulses create an oscillatory temperature rise (a few Kelvins) on the surface which induces changes in the thermoreflectivity that can be related to thermal properties of the underlying layers. In order to facilitate capturing the changes in the surface thermoreflectvity, all samples were coated with an 80 $\mathrm{nm}$ of ruthenium transducer. The pump and probe beams, after passing through a 10X objective, have spot sizes of $\sim 20$ and $\sim 10 \mu \mathrm{m}$ on the surface of the sample, respectively. The thermal model that relates the changes in the thermoreflectivity to the thermal properties of the underlying layers, requires knowledge of volumetric heat capacity, thermal conductivity and film thickness. We use a volumetric heat capacity of 2.96 and $1.64 \mathrm{MJ} \mathrm{m}^{-3} \mathrm{~K}^{-1}$ and thermal conductivity of 54 and $145 \mathrm{~W} \mathrm{~m}^{-1} \mathrm{~K}^{-1}$ for ruthenium and silicon, respectively. The thickness of each layer is determined via TEM. 
Simulation approach. Due to a lack of interatomic potential that captures the coordination number variations across the composition range in $\mathrm{SiTe}$ alloy, we use $a-\mathrm{Si}, a-\mathrm{SiO}_{2}$, and $a-\mathrm{Si}_{20} \mathrm{Te}_{80}$ compositions for comparison. The interatomic potentials used for these compositions are Stilinger-Weber (SW) for $a$-Si, Beest-Kramer-van Santen ${ }^{33,41}$ (BKS) for $a-\mathrm{Si}^{127} \mathrm{O}_{2}$, and force constants derived from $a b$-initio calculations for $a$ $\mathrm{Si}_{20} \mathrm{Te}_{80}$.

For our ab-initio density functional calculations, we have used the projector augmented wave (PAW) approach as implemented in $\operatorname{VASP}^{42,43}$. The exchange and correlation energies are represented in the generalized gradient approach (GGA) using the Perdue-Burke-Ernzerhof (PBE) exchangecorrelation functional. Given that we are investigating glassy materials, it is important to consider a large enough supercell to approximate these disordered materials. We found that 300 atom supercells were sufficient to limit interaction with mirror images of atoms due to the periodic boundary conditions. For $\mathrm{Si}_{20} \mathrm{Te}_{80}$, there is strong evidence that $\mathrm{Si}$ prefers a tetrahedral configuration bonded to four $\mathrm{Te}$ atoms $\left(\mathrm{SiTe}_{4}\right)^{44}$. Using the Packmol routine ${ }^{45}$ as implemented in QuantumATK ${ }^{46}$, we create ten different initial atomic configurations by packing 60 of these $\mathrm{SiTe}_{4}$ units in a supercell. The volume of the supercell is determined by the measured mass density ${ }^{47}$ (5.08 $\mathrm{g} / \mathrm{cm}^{3}$ ). Structural relaxation of the system is then performed to reach the minimum energy configuration. The energies of the final structures show some distribution which is to be expected for a glassy material that can have numerous closely spaced metastable energy states. To examine the impact of bond arrangement on the alloy properties, we also generated supercells where the initial atomic arrangement was random and there was no explicit chemical ordering. Structural relaxation was performed on ten different configurations for these atomic networks as well. For both the chemically ordered and physical bonding networks, we use the relaxed configuration with the lowest energy as the basis for subsequent ab-initio molecular dynamic (MD) simulations. For $\mathrm{Si}_{20} \mathrm{Te}_{80}$, we ran 8 ps and 20 ps simulation runs at $\mathrm{T}=500 \mathrm{~K}$ with a fs time step. To ensure our analysis does not suffer from any thermalization effects, we discard data from the first picosecond in our trajectory analysis. From the trajectory of the MD run, we can then calculate the vibrational density of states for $\mathrm{Si}_{20} \mathrm{Te}_{80}$.

The vibrational density of states for a supercell with $N$ atoms can be written as:

$$
S(v)=\frac{2}{k_{B} T} \sum_{i=1}^{N} \sum_{\alpha=x, y, z} m_{i} s_{i}^{\alpha}(v)
$$

where $v$ is the frequency, $\mathrm{m}_{i}$ is the mass of atom $i$, and $s_{i}^{\alpha}$ is the spectral density of atom $i$ in the Cartesian direction $\alpha$. The vibrational density of states is determined by the sum over all $N$ atoms and over all Cartesian directions. The spectral density can be written in terms of the square of the Fourier transform of the atomic velocity ${ }^{48}$.

$$
s_{i}^{\alpha}(v)=\frac{\left|\int_{0}^{t_{\max }} d t^{\prime} v_{i}^{\alpha}\left(t^{\prime}\right) e^{-i 2 \pi v t^{\prime}}\right|^{2}}{t_{\max }}
$$

where $\mathrm{t}_{\max }$ is the maximum time in the MD trajectory. The current approach is efficient and can be shown to be equivalent to the more computationally intensive calculation of the vibrational density of states based on the velocity auto-correlation function.

While molecular dynamics simulations can provide insight into the vibrational density of states at a given temperature, they can not provide us with direct information on the vibrational eigenvector and the degree of localization for each mode. To complement the MD simulations, we have also performed real-space force constant calculations on the fully relaxed 300 atom supercell for $\mathrm{Si}_{20} \mathrm{Te}_{80}$ using VASP to determine the forces on perturbed supercells $(0.01 \AA$ displacement $)$ and Phonopy to extract the force constants and dynamical matrix $^{49}$. For our amorphous $\mathrm{Si}_{20} \mathrm{Te}_{80}$ supercell, the lack of any crystalline symmetry leads to 1800 separate supercell calculations to determine the appropriate forces. The vibrational density of states was calculated using the dynamical matrix and shows good agreement with the predicted vibrational density of states from the long 20 ps molecular dynamics simulation. This is an important consistency check between the two separate approaches to predict vibrational properties.

\section{DATA AVAILABILITY.}

The data that support the findings of this study are available from the corresponding author upon reasonable request.

\section{ACKNOWLEDGMENTS}

We appreciate support from Western Digital Technologies, Inc. The first author would like to thank Rouzbeh Rastgar, Jason Larkin, Ashutosh Giri, and Julian Gale for helpful conversations. This work was supported in part by the NSF I/ UCRC on Multi-functional Integrated System Technology (MIST) Center IIP-1439644, IIP-1439680, and IIP-1738752.

\section{AUTHOR CONTRIBUTIONS}

K.A., D.A.S., J.N., J.T.G., M.K.G., and P.E.H designed the experiment. J.N. and J.C.R. made the samples. K.A., J.T.G., and D.H.O. performed the experiments. K.A., D.A.S. performed the simulations. K.A., D.A.S. and P.E.H wrote the manuscript.

\section{COMPETING INTERESTS}

The authors declare no competing interests.

\section{ADDITIONAL INFORMATION}

Supplementary information is available for this paper at 
Correspondence and requests for materials should be addressed to P.E.H.

\section{REFERENCES}

${ }^{1}$ John C Duda, Patrick E Hopkins, Yang Shen, and Mool C Gupta. Exceptionally low thermal conductivities of films of the fullerene derivative pcbm. Physical review letters, 110(1):015902, 2013.

${ }^{2}$ Li-Dong Zhao, Shih-Han Lo, Yongsheng Zhang, Hui Sun, Gangjian Tan, Ctirad Uher, Christopher Wolverton, Vinayak P Dravid, and Mercouri G Kanatzidis. Ultralow thermal conductivity and high thermoelectric figure of merit in snse crystals. Nature, 508(7496):373, 2014.

${ }^{3}$ Ashutosh Giri, Alexander Z Chen, Alessandro Mattoni, Kiumars Aryana, Depei Zhang, Xiao Hu, Seung-Hun Lee, Joshua J Choi, and Patrick E Hopkins. Ultralow thermal conductivity of two-dimensional metal halide perovskites. Nano Letters, 2020.

${ }^{4}$ David G Cahill and RO Pohl. Heat flow and lattice vibrations in glasses. Solid State Communications, 70(10):927-930, 1989.

${ }^{5}$ Philip B Allen and Joseph L Feldman. Thermal conductivity of glasses: Theory and application to amorphous si. Physical review letters, 62(6):645, 1989.

${ }^{6}$ David G Cahill, Susan K Watson, and Robert O Pohl. Lower limit to the thermal conductivity of disordered crystals. Physical Review B, 46(10):6131, 1992.

${ }^{7}$ Matthias T Agne, Riley Hanus, and G Jeffrey Snyder. Minimum thermal conductivity in the context of diffuson-mediated thermal transport. Energy \& Environmental Science, 11(3):609-616, 2018.

${ }^{8}$ Zhen Chen and Chris Dames. An anisotropic model for the minimum thermal conductivity. Applied Physics Letters, 107(19):193104, 2015.

${ }^{9}$ Catalin Chiritescu, David G Cahill, Ngoc Nguyen, David Johnson, Arun Bodapati, Pawel Keblinski, and Paul Zschack. Ultralow thermal conductivity in disordered, layered wse ${ }_{2}$ crystals. Science, 315(5810):351-353, 2007.

${ }^{10}$ Wee-Liat Ong, Evan S O’Brien, Patrick SM Dougherty, Daniel W Paley, C Fred Higgs III, Alan JH McGaughey, Jonathan A Malen, and Xavier Roy. Orientational order controls crystalline and amorphous thermal transport in superatomic crystals. Nature materials, 16(1):83-88, 2017.

${ }^{11}$ Jeffrey L Braun, Christina M Rost, Mina Lim, Ashutosh Giri, David H Olson, George N Kotsonis, Gheorghe Stan, Donald W Brenner, Jon-Paul Maria, and Patrick E Hopkins. Charge-induced disorder controls the thermal conductivity of entropy-stabilized oxides. Advanced Materials, 30(51):1805004, 2018.

${ }^{12}$ Marc G Ghossoub, Jung-Hyun Lee, Oksen T Baris, David G Cahill, and Sanjiv Sinha. Percolation of thermal conductivity in amorphous fluorocarbons. Physical Review B, 82(19):195441, 2010.

${ }^{13}$ Sean W King, Jeff Bielefeld, Guanghai Xu, William A Lanford, Yusuke Matsuda, Reinhold H Dauskardt, Namjun Kim, Donald Hondongwa, Lauren Olasov, Brian Daly, et al. Influence of network bond percolation on the thermal, mechanical, electrical and optical properties of high and low-k a-sic: H thin films. Journal of Non-Crystalline Solids, 379:67-79, 2013.

${ }^{14}$ Jeffrey L Braun, Sean W King, Ashutosh Giri, John T Gaskins, Masanori Sato, Takemasa Fujiseki, Hiroyuki Fujiwara, and Patrick E Hopkins. Breaking network connectivity leads to ultralow thermal conductivities in fully dense amorphous solids. Applied Physics Letters, 109(19):191905, 2016.

${ }^{15} \mathrm{~J}$. C. Phillips. Topology of covalent non-crystallien solids i: Short-range order in chalcogenide alloys. Journal of Non-Crystalline Solids, 34:153, 1979.

${ }^{16}$ Xingwei Feng, WJ Bresser, and P Boolchand. Direct evidence for stiffness threshold in chalcogenide glasses. Physical review letters, 78(23):4422, 1997.

${ }^{17}$ D Selvanathan, WJ Bresser, P Boolchand, and B Goodman. Thermally reversing window and stiffness transitions in chalcogenide glasses. Solid state communications, 111(11):619-624, 1999.

${ }^{18}$ Hugo M Flores-Ruiz, Gerardo G Naumis, and JC Phillips. Heating through the glass transition: a rigidity approach to the boson peak. Physical Review $B, 82(21): 214201,2010$.

${ }^{19} \mathrm{CH}$ Thomsen, Holger T Grahn, Humphrey J Maris, and Jan Tauc. Surface generation and detection of phonons by picosecond light pulses. Physical Review B, 34(6):4129, 1986.

${ }^{20}$ J. L. Braun, C. H. Baker, A. Giri, M. Elahi, K. Artyushkova, T. E. Beechem, P. M. Norris, Z. C. Leseman, J. T. Gaskins, and P. E. Hopkins. Size effects on the thermal conductivity of amorphous silicon thin films. Physical Review B, 93:140201, 2016.

${ }^{21}$ Bruno Bureau, Sylvain Danto, Hong Li Ma, Catherine Boussard-Plédel, Xiang Hua Zhang, and Jacques Lucas. Tellurium based glasses: A ruthless glass to crystal competition. Solid State Sciences, 10(4):427-433, 2008.

${ }^{22}$ Cho Yen Ho, Reginald W Powell, and Peter E Liley. Thermal conductivity of the elements. Journal of Physical and Chemical Reference Data, 1(2):279-421, 1972.

${ }^{23}$ Hua Peng, Nicholas Kioussis, and Derek A Stewart. Anisotropic lattice thermal conductivity in chiral tellurium from first principles. Applied Physics Letters, 107(25):251904, 2015.

${ }^{24}$ Siqi Lin, Wen Li, Zhiwei Chen, Jiawen Shen, Binghui Ge, and Yanzhong Pei. Tellurium as a high-performance elemental thermoelectric. Nature communications, 7(1):1-6, 2016.

${ }^{25}$ David G Cahill and Robert O Pohl. Lattice vibrations and heat transport in crystals and glasses. Annual review of physical chemistry, 39(1):93-121, 1988.

${ }^{26}$ Philip B Allen and Joseph L Feldman. Thermal conductivity of disordered harmonic solids. Physical Review B, 48(17):12581, 1993.

${ }^{27}$ Hamid Reza Seyf and Asegun Henry. A method for distinguishing between propagons, diffusions, and locons. Journal of Applied Physics, 120(2):025101, 2016.

${ }^{28} \mathrm{~J}$. Moon and A. J. Minnich. Sub-amorphous thermal conductivity in amorphous heterogeneous nanocomposites. RSC Advances, 6:105154, 2016.

${ }^{29}$ J. Chen, G. Zhang, and B. Li. Remarkable reduction of thermal conductivity in silicon nanotubes. Nano Letters, 10:3978, 2010.

${ }^{30} \mathrm{~L}$. Yang, N. Yang, and B. Li. Extreme low thermal conductivity in nanoscale 3d phononic crystals with spherical pores. Nano Letters, 14:1734, 2014.

${ }^{31}$ Philip B Allen, Joseph L Feldman, Jaroslav Fabian, and Frederick Wooten. Diffusons, locons and propagons: Character of atomie yibrations in amorphous si. Philosophical Magazine B, 79(11-12):1715-1731, 1999.

${ }^{32}$ Mihai Popescu. Non-crystalline chalcogenides. Kluwer Academic Publishers, Dordrecht,, 2001.

${ }^{33}$ Jason M Larkin and Alan JH McGaughey. Thermal conductivity accumulation in amorphous silica and amorphous silicon. Physical Review B, 89(14):144303, 2014.

${ }^{34}$ Jacob Philip and KN Madhusoodanan. Percolation threshold of thermal conduction in a $\mathrm{x}$ iv $\mathrm{b} 1-\mathrm{x}$ vi chalcogenide semiconducting glasses. Physical Review B, 38(6):4127, 1988.

${ }^{35}$ Jason Lonergan, Charmayne Smith, Devon McClane, and Kathleen Richardson. Thermophysical properties and conduction mechanisms in asxse $1-\mathrm{x}$ chalcogenide glasses ranging from $\mathrm{x}=0.2$ to 0.5 . Journal of Applied Physics, 120(14):145101, 2016.

${ }^{36}$ Lanhua Wei, Mark Vaudin, Cheol Song Hwang, Grady White, Jason Xu, and Andrew J Steckl. Heat conduction in silicon thin films: Effect of microstructure. Journal of materials research, 10(8):1889-1896, 1995.

${ }^{37}$ Sheng-Nan Zhang, Jian He, Tie-Jun Zhu, Xin-Bing Zhao, and Terry M Tritt. Thermal conductivity and specific heat of bulk amorphous chalcogenides ge20te80- xsex $(\mathrm{x}=0,1,2,8)$. Journal of Non-Crystalline Solids, 355(2):79-83, 2009.

${ }^{38}$ António Pereira Gonçalves, Elsa Branco Lopes, Olivier Rouleau, and Claude Godart. Conducting glasses as new potential thermoelectric materials: the cu-ge-te case. Journal of Materials Chemistry, 20(8):1516-1521, 2010.

${ }^{39}$ Roberto Fallica, Enrico Varesi, Luca Fumagalli, Simona Spadoni, Massimo Longo, and Claudia Wiemer. Effect of nitrogen doping on the thermal conductivity of gete thin films. physica status solidi (RRL)-Rapid Research Letters, 7(12):1107-1111, 2013.

${ }^{40}$ Ronald J Warzoha, Brian F Donovan, Nicholas T Vu, James G Champlain, Shawn Mack, and Laura B Ruppalt. Nanoscale thermal transport in amorphous and crystalline gete thin-films. Applied Physics Letters, 115(2):023104, 2019.

${ }^{41}$ BWH Van Beest, Gert Jan Kramer, and RA Van Santen. Force fields for silicas and aluminophosphates based on ab initio calculations. Physical Review Letters, 64(16):1955, 1990.

${ }^{42} \mathrm{G}$. Kresse and J. Furthmuller. Efficient iterative schemes for ab initio 
total-energy calculations using a plane-wave basis set. Physical Review $B, 54: 11169,1996$.

${ }^{43}$ G. Kresse and J. Furthmuller. Efficiency of ab-initio total energy calculations for metals and semiconductors using a plane-wave basis set. J. Computer. Mater. Sci., 15:6, 1996.

${ }^{44}$ M. Micoulaut, K. Gunasekera, S. Ravindren, and P. Boolchand. Quantitative measure of tetrahedral-sp ${ }^{3}$ geometries in amorphous phase-change alloys. Physical Review B, 90:90, 2014.

${ }^{45}$ L. Martinez, R. Andrade, E. G. Birgin, and J. M. Martinez. Packmol: A package for building initial configurations for molecular dynamic simulations. Journal of Computational Chemistry, 30:2157, 2009.

${ }^{46}$ Søren Smidstrup, Troels Markussen, Pieter Vancraeyveld, Jess Wellendorff, Julian Schneider, Tue Gunst, Brecht Verstichel, Daniele Stradi, Petr A
Khomyakov, Ulrik G Vej-Hansen, et al. Quantumatk: An integrated platform of electronic and atomic-scale modelling tools. J. Phys: Condens. Matter, 32:015901, 2020.

${ }^{47}$ K. Tsunetomo, R. Shimizu, T. Imura, and Y. Osaka. Exafs and x-ray diffraction studies on the local structure of sputter-deposited amorphous $\mathrm{si}_{x} \mathrm{te}_{1-x}$ alloys. Journal of Non-Crystalline Solids, 116:262, 1990.

${ }^{48}$ S.-T. Lin, M. Blanco, and W. A. Goddard. The two-phase model for calculating thermodynamic properties of liquids from molecular dynamics: Validation for the phase diagram of lennard-jones fluids. Journal of Chemical Physics, 119:11792, 2003.

${ }^{49} \mathrm{~A}$ Togo and I Tanaka. First principles phonon calculations in materials science. Scr. Mater., 108:1-5, Nov 2015. 

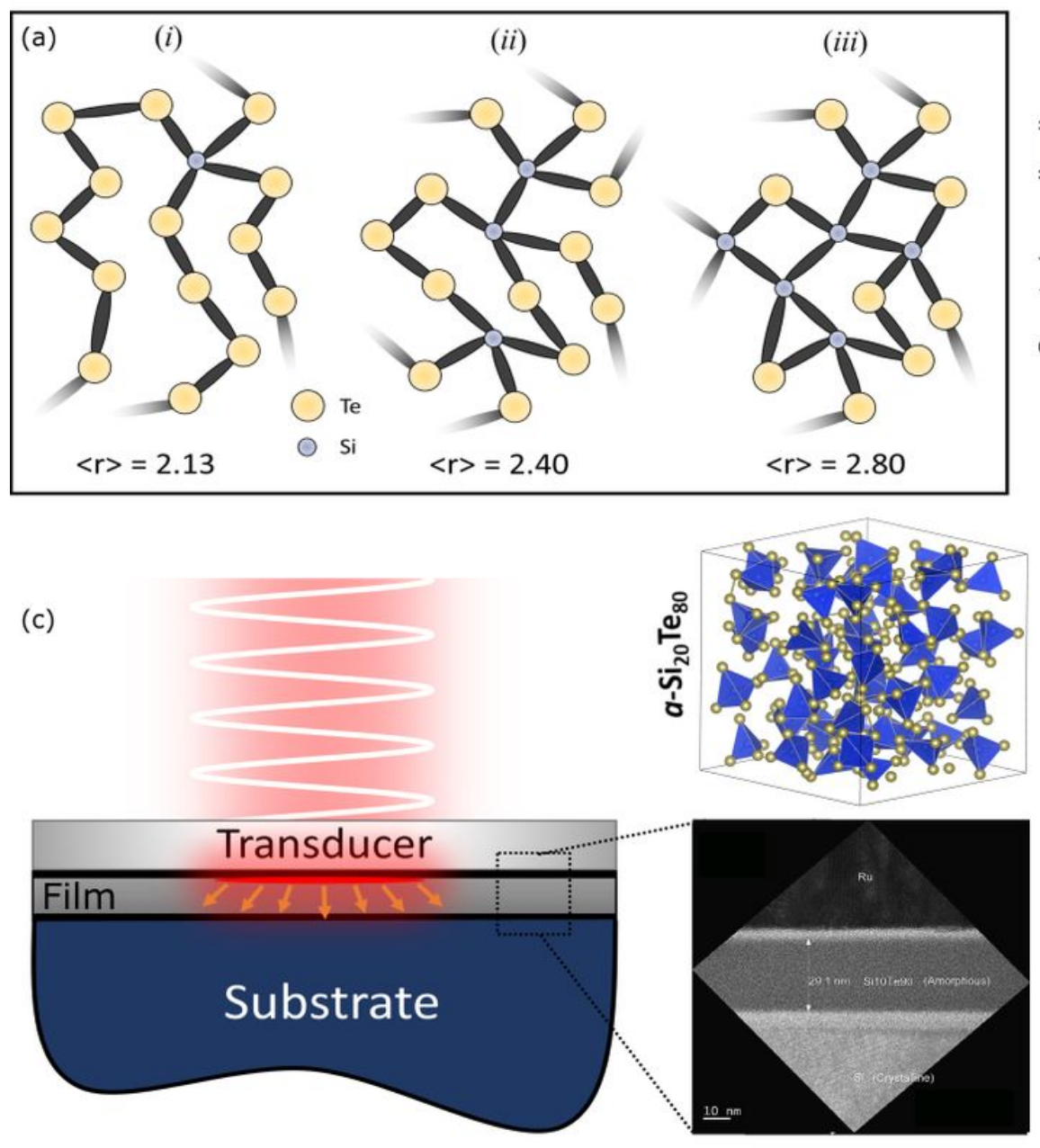
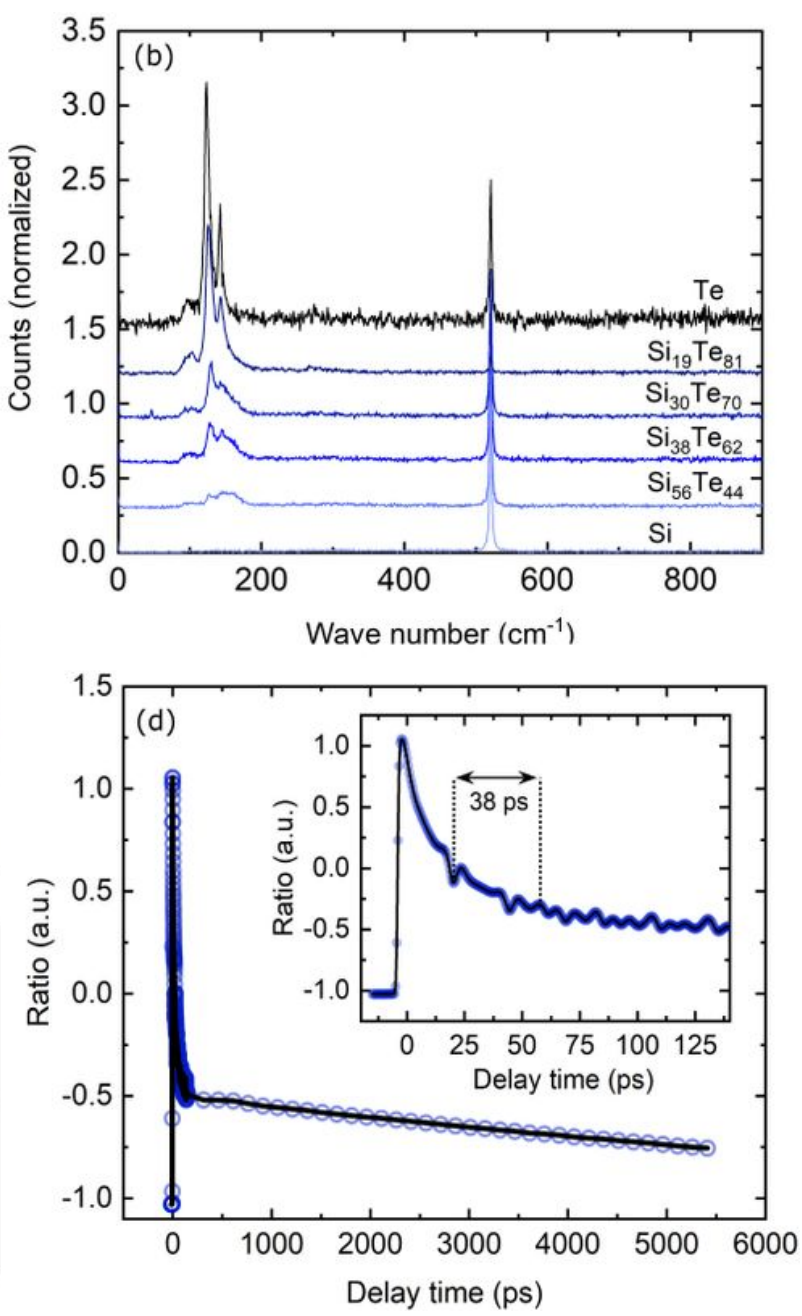

Figure 1

(a) Schematics of amorphous networks with a total of 15 atomic sites for different Te to Si ratios representing various topological regimes: (i) under-constrained network, (ii) stress-free network, and (iii) over-constrained network. (b) Raman spectra for SiTe at different Te concentrations. The sharp silicon peak is the effect of the substrate and is not associated with the thin film properties. (c) Illustration of TDTR measurement geometry, measurement technique and the corresponding layers studied, (d) Representative TDTR data for $40 \mathrm{~nm}$ thick a-Si19Te81. The inset shows the picosecond acoustic measurements where the distance between the troughs and the peaks in the measured TDTR data are related to the time it takes for acoustic waves traveling at the speed of sound to transverse the aSi19Te81 film. 

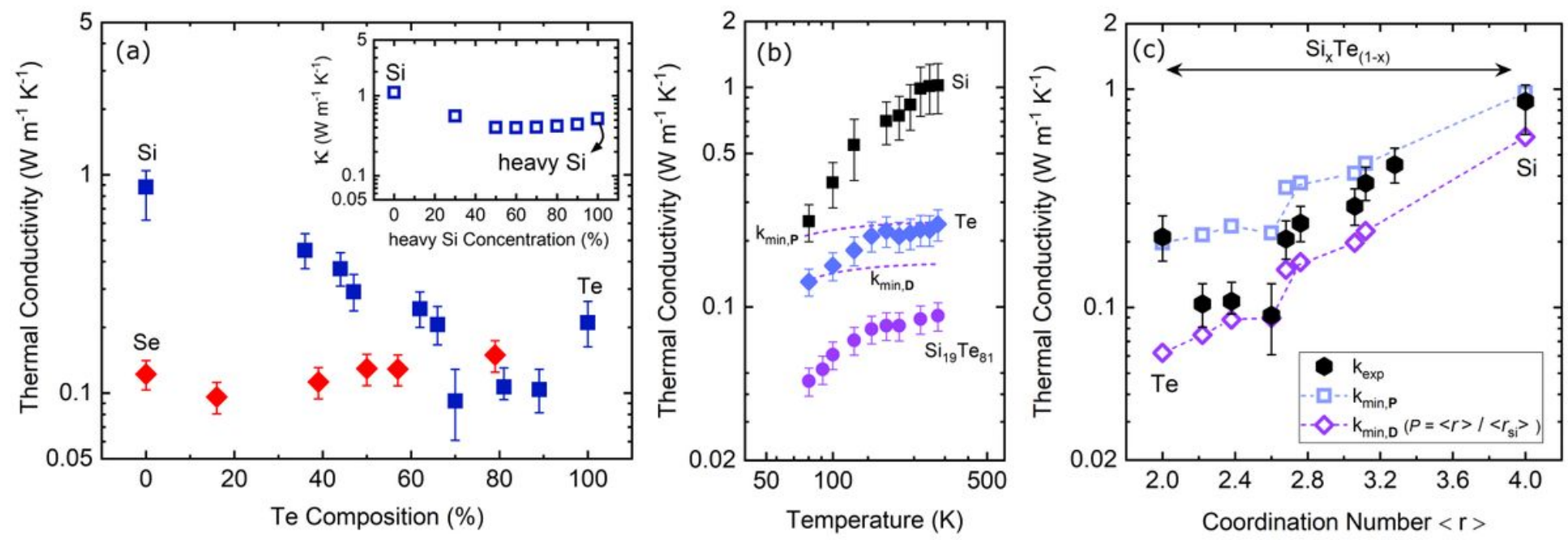

Figure 2

(a) The measured thermal conductivity as a function of tellurium concentration in amorphous SiTe and SeTe. The inset shows the NEMD simulation results for thermal conductivity of Si ( $28 \mathrm{u}$ ), heavy Si (127 u), and their alloy. (b) Thermal conductivity of amorphous silicon, tellurium, and Si19Te81 as a function of temperature. For comparison, $62 \mathrm{~nm}$ WSe2 data are included from Ref9. (c) Thermal conductivity of SiTe as a function of coordination number measured by TDTR (solid hexagons), their corresponding minimum limit (hollow squares), and the diffuson-mediated thermal conductivity (hollow diamonds).
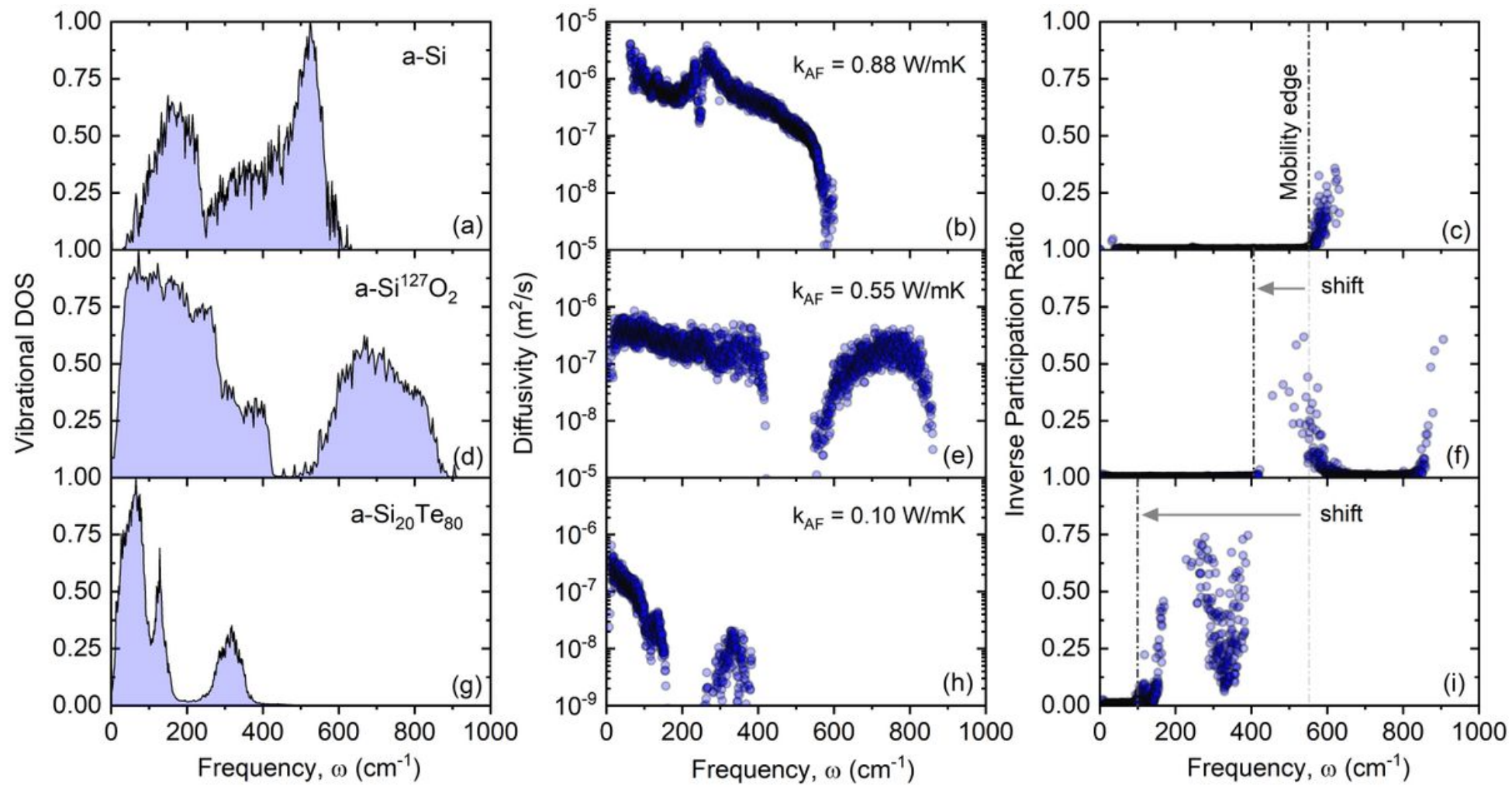

Figure 3 
$(a, d, g)$ Vibrational density of states (DOS), (b,e,h) modes diffusivity, and (c,f,i) inverse participation ratio (IPR) for a-Si, a-Si12702, and a-Si20Te80, respectively. The corresponding thermal conductivity are given for each system. The shift in the mobility edge with respect to the a-Si is depicted by an arrow.
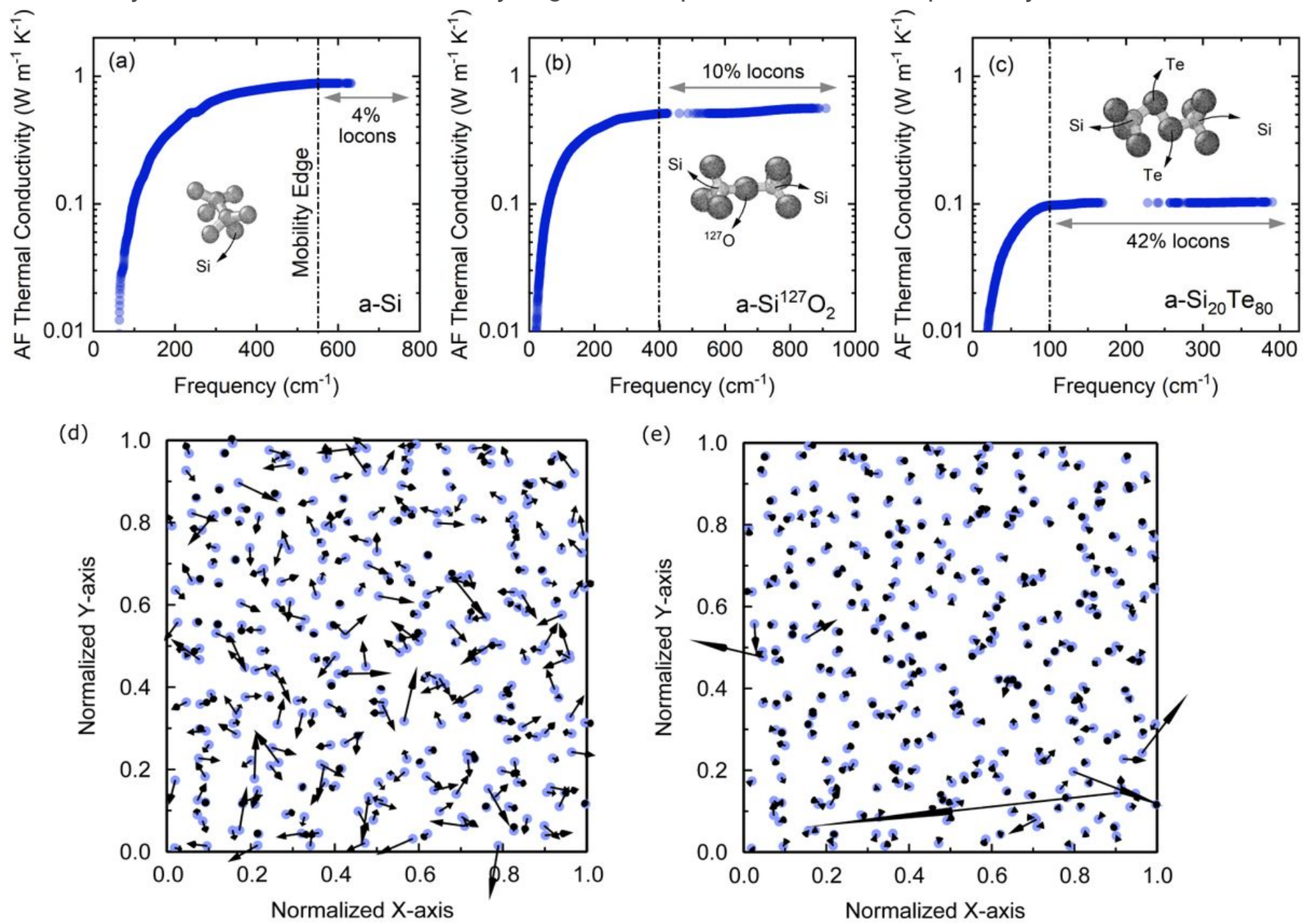

(f)

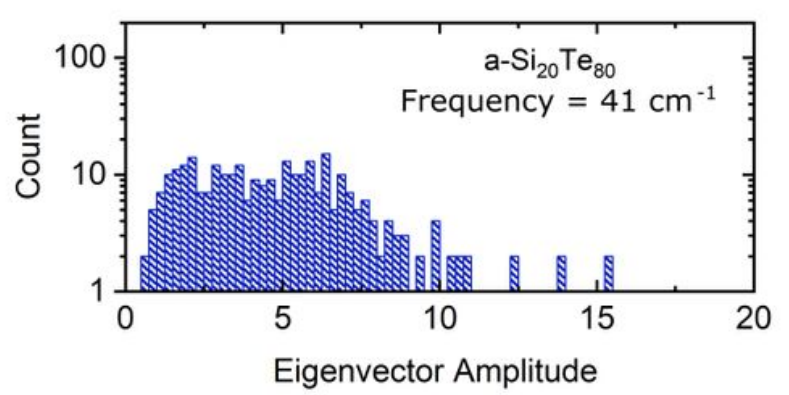

(g)

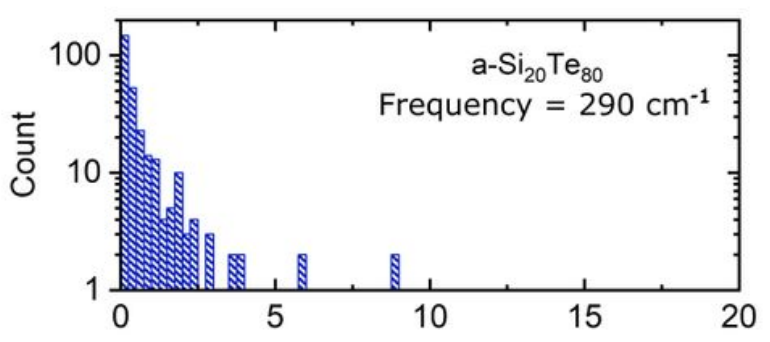

Eigenvector Amplitude

Figure 4

Allen-Feldman thermal conductivity accumulation as a function of vibrational mode frequency for (a) awith inset showing Si-Si bond between two tetrahedrons, (b) a-Si12702 with inset showing Si-1270-Si bond between two tetrahedrons, and (c) a-Si20Te80 with inset showing Si-Te-Te-Si bond between two tetrahedrons. (d,e) Visualization of delocalized $(41 \mathrm{~cm}-1)$ and localized $(290 \mathrm{~cm}-1)$ vibrational modes on either side of the mobility edge in a-Si20Te80. The few high amplitude eigenvectors in localized frequency are an indication of strong localization showing the energy associated with these modes are 
confined in a small geometric region. ( $f, e)$ Histogram indicating the population of modes based on their eigenvector amplitude at delocalized and localized frequencies.

\section{Supplementary Files}

This is a list of supplementary files associated with this preprint. Click to download.

- supplement.pdf 